${ }^{1}$ Departamento de Medicina Intensiva. Pontificia Universidad Católica de Chile.

${ }^{2}$ Unidad de Pacientes Críticos. Hospital Clínico de la Universidad de Chile. Santiago. Chile.

Recibido el 31 de marzo de 2014, aceptado el 28 de octubre de 2014.

Conflictos de intereses: nada que declarar.

Correspondencia a:

Dr. Guillermo Bugedo Marcoleta 367, Santiago, Chile. Fax: 56226641329

Zip code 6510260 gbugedo@gmail.com

\title{
Manejo del potencial donante cadáver
}

\author{
GUILLERMO BUGEDO ${ }^{1}$, SEBASTIÁN BRAVO ${ }^{1}$, \\ CARLOS ROMERO ${ }^{2}$, RICARDO CASTRO ${ }^{1}$
}

\section{Management of the potential organ donor}

\begin{abstract}
Solid organ transplantation is limited by donor availability. The loss of brain function produces hemodynamic, respiratory, hormonal and metabolic changes that lead to hypotension and organ dysfunction. Management of a potential donor is similar to any critically ill patient. Cardiovascular stability and protective ventilatory support must be pursued, aimed at minimizing the local and systemic inflammatory response that is triggered by brain death. There is no consensus on protocols for hormonal supplementation. The administration of vasopressin analogues and steroids may be beneficial under certain conditions. Appropriate medical management helps to optimize the function of different organs prior to transplantation. This may increase the number of harvested organs and improve their functional outcome in the recipient.
\end{abstract}

(Rev Med Chile 2014; 142: 1584-1593)

Key words: Brain death; Living donors; Organ transplantation; Tissue donors.

E 1 trasplante de órganos sólidos (riñón, hígado, páncreas, corazón y pulmón) está muy limitado por la disponibilidad de donantes $^{1,2}$. La muerte encefálica es una condición catastrófica con marcada inestabilidad circulatoria, que si no es manejada en forma oportuna y adecuada puede llevar al deterioro de los órganos antes de su procuramiento ${ }^{3,4}$. El apropiado manejo del potencial donante antes y después del diagnóstico de muerte encefálica puede mejorar el número y calidad de los órganos procurados (Tablas 1 y 2$)^{5}$. Lamentablemente, aún en la actualidad, muchos potenciales donantes fallecen por un manejo hemodinámico o respiratorio inadecuado.

La mayor parte de los órganos trasplantados proviene de pacientes que caen en muerte encefálica secundaria a trauma, enfermedad cerebrovascular, encefalopatía anóxica, algunos tumores cerebrales primarios, etc. La gran demanda de pacientes en espera de órganos ha llevado a la expansión de los criterios clásicos para donación (criterio expandido), incluyendo ahora pacientes de edades extremas (menos de 5 años y mayor de 60 años), con comorbilidades, con insuficiencia renal, e incluso hasta con alguna infección bacte- riana. Los injertos “marginales” tienen mayor tasa de disfunción primaria del injerto y muerte, pero su definición y manejo apropiado es importante pues hay receptores que se beneficiarán aun de órganos procurados en un paciente con estas características. Como siempre, la evaluación de cada caso en particular, considerando la mortalidad del paciente en espera, es fundamental para una correcta toma de decisiones ${ }^{1,6,8}$. En otros centros, ya existe amplia experiencia en la donación de órganos después de la muerte o paro cardiaco, pero este tema excede los objetivos de la presente revisión ${ }^{9,10}$.

El presente manuscrito es una propuesta de manejo del potencial donante cadáver desde la perspectiva del intensivista y principalmente referido a la donación de órganos perfundidos: corazón, pulmón, hígado, páncreas y riñón. $\mathrm{Su}$ objetivo es alertar a la comunidad médica respecto de la necesidad de optimizar la función de estos órganos previo al trasplante, realizando una detección precoz de los potenciales donantes y siendo agresivos en la prevención y manejo de sus complicaciones. Muchos donantes son procurados en hospitales donde no se cuenta con médicos 
Manejo del potencial donante de órganos - G. Bugedo et al

\section{Tabla 1. Manejo General del potencial donante cadáver}

a) Manejo en Unidad de Pacientes Críticos o similar

b) Mantener estabilidad circulatoria

c) Sostener ventilación protectora y tratar hipoxemia

d) Mantener el equilibrio ácido-básico, así como los niveles de $\mathrm{Na}+, \mathrm{K}+$, calcio y fósforo en rangos fisiológicos

e) Procurar un ambiente térmico neutral con $\mathrm{T}^{\circ}$ corporales $>35^{\circ} \mathrm{C}$ en forma continua

Tabla 2. Monitorización mínima en paciente potencial donante de órganos
a) Electrocardiografía continua
b) Temperatura axilar cada 1-2 $\mathrm{h}$
c) Presión arterial directa
d) Catéter venoso central y presión venosa central (PVC)
e) Sonda vesical y diuresis horaria
f) Oxímetro de pulso

entrenados en el manejo de pacientes críticos y menos aún en este subgrupo especial de enfermos, cuyo desenlace es inevitable pero que se manejan tanto o más activamente que un paciente crítico pero con mejores expectativas vitales. Para más detalles con respecto a epidemiología, legislación, manejo anestésico y quirúrgico, inmunosupresión, entre otros tópicos, sugerimos referirse a otros documentos, entre ellos las Guías Clínicas de la Sociedad Chilena de Trasplante ${ }^{11}$.

\section{Muerte encefálica}

Debemos partir mencionando el concepto de muerte encefálica. La muerte de un paciente, entendida como la cesación permanente del funcionamiento del organismo de un ser humano particular, puede ser definida en términos clínicos, biológicos, bioquímicos e incluso espirituales, cuyo alcance excede por lejos el objetivo del presente documento. Junto con cambios importantes y continuos en la definición y nomenclatura de muerte, tenemos dos criterios que actualmente comparten el mayor consenso, a saber:
1) La pérdida permanente de la función cardiorrespiratoria, que es el criterio utilizado tradicionalmente, $y$

2) La pérdida total e irreversible de la función del encéfalo completo.

Vale decir, existe consenso que sin función encefálica no es posible sostener la función cardiorrespiratoria. Esta aceptación en la comunidad científica debe ser claramente comunicado a la familia, y con especial cuidado en la precisión y prudencia frente a un potencial donante.

Para contexto y consideraciones legales con respecto de la muerte encefálica en Chile, sugerimos revisar la jurisprudencia respectiva (Sentencia del Tribunal Constitucional rol 220 del 13 de agosto de 1995, la Ley 19.451 de 1996, el Decreto Supremo No 656 de 1997 y la Resolución Exenta No 48 del Ministerio de Salud de 2000). Sólo mencionaremos lo medular del fallo del Tribunal Constitucional que, en su artículo 15 señala: "Que examinados los informes científicos antes señalados (...) se llega a la conclusión de que la abolición total e irreversible de todas las funciones encefálicas constituye la muerte real, definitiva, unívoca e inequivoca del ser humano"12.

\section{Manejo hemodinámico del potencial donante cadáver}

El cese de la función cerebral produce alteraciones hormonales, metabólicas, circulatorias y respiratorias, que llevan a la pérdida de los mecanismos regulatorios de los sistemas cardiovascular y respiratorio ${ }^{13}$. La hipotensión es uno de los problemas más frecuentes en los potenciales donantes, siendo una resultante de hipovolemia, vasoplejia, disfunción cardiaca y trastornos hormonales (Figura 1). Más importante, la hipotensión está asociada a una mayor incidencia de necrosis tubular aguda y menor sobrevida a largo plazo de los injertos perfundibles ${ }^{3,5,14,16}$. Además, entre 25 a $40 \%$ de los pacientes en muerte encefálica presentan disfunción cardiaca en la ecocardiografía, que puede ser revertida con un buen manejo de fluidos $\mathrm{y}$ dosis bajas de drogas vasoactivas e inótropos ${ }^{17,18}$.

En general, el manejo hemodinámico del potencial donante es la continuación del manejo previo del paciente crítico, pero con un cambio en las metas. Ya no se pretende controlar o revertir la enfermedad de base (que llevó a la muerte ce- 


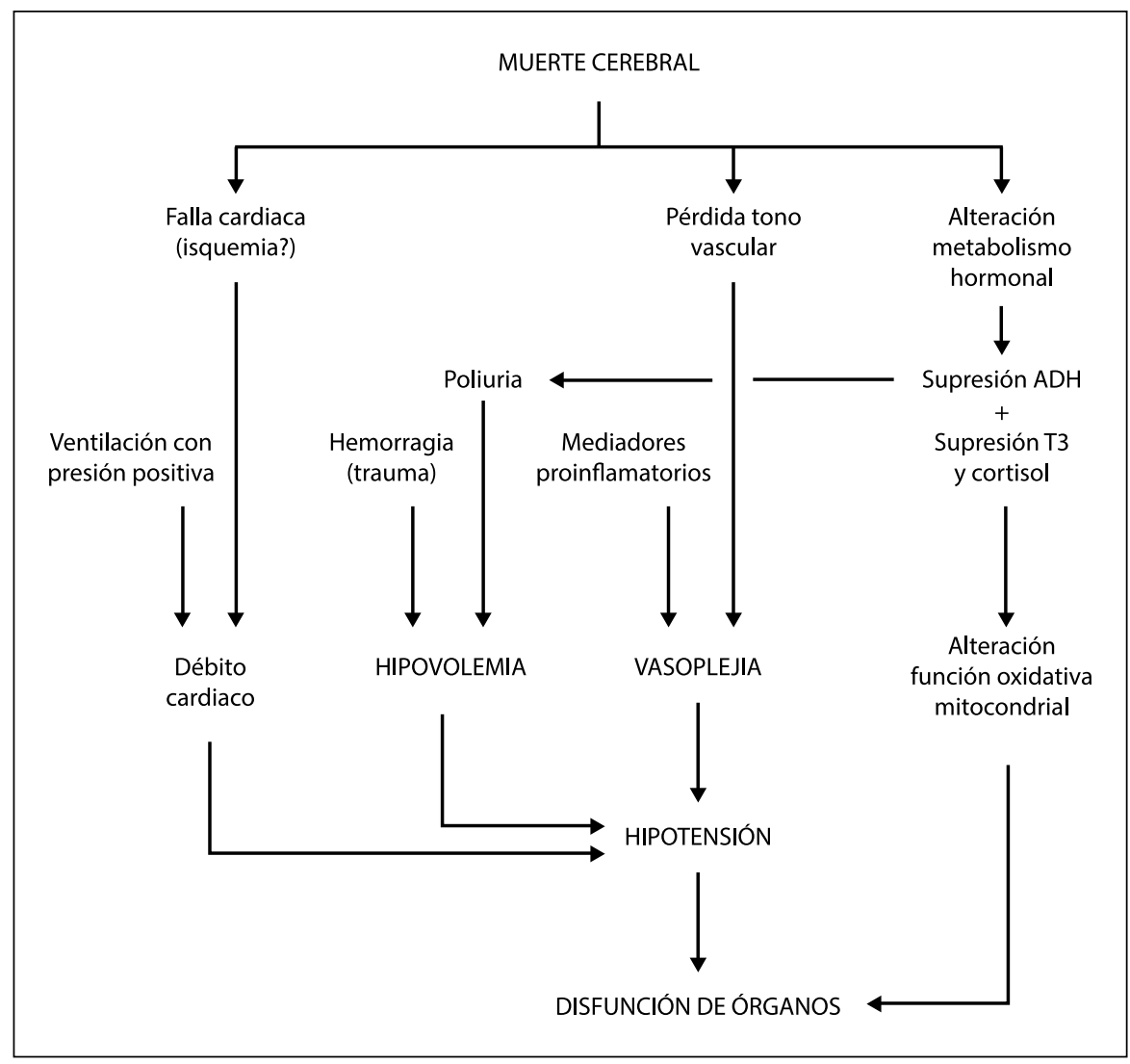

Figura 1. Mecanismos de hipotensión y disfunción de órganos durante la muerte cerebral. Abreviaciones: $A D H$, hormona antidiurética; T3, triyodotironina. rebral), sino mantener la perfusión y homeostasis tisular.

La aproximación inicial del potencial donante con hipotensión contempla una evaluación de la precarga y la reposición del volumen intravascular con el uso de cristaloides balanceados (p.e. suero Ringer lactato) (Figura 2 y Tabla 3). La precarga es una variable de difícil valoración en la clínica. La variación de la presión de pulso es, en general, superior a las mediciones estáticas de presión venosa central (PVC) o de capilar pulmonar en predecir la respuesta al aporte de fluidos ${ }^{19}$. En un estudio reciente en 20 potenciales donantes, la mitad de ellos tenía una variación en la presión de pulso $>13 \%$, sugerente de hipovolemia, lo cual se asoció a un aumento de mediadores proinflamatorios $^{20}$. El uso del catéter de arteria pulmonar (Swan-Ganz) ha disminuido consistentemente en los últimos años en los pacientes críticos. No obstante, podría ser útil en pacientes con inestabilidad hemodinámica si no se dispone de otras herramientas menos invasivas y que requieren entrenamiento como la ecocardiografía. Esta es una técnica mínimamente invasiva cada vez más en uso, que en potenciales donantes de órganos sólidos y mayormente de corazón es fundamental para evaluar y optimizar la función cardiaca, la precarga y la postcarga ${ }^{17}$.

\section{Tabla 3. Manejo de la hipotensión}

a) Reposición de volumen en base a cristaloides balanceados (suero Ringer lactato) hasta lograr una PVC 4-8 $\mathrm{mmHg}$ (o $\triangle \mathrm{PP}<13 \%$ ), y presión arterial sistólica (PAS) $\geq 100 \mathrm{mmHg}$ y media (PAM) $\geq 70-80 \mathrm{mmHg}$

b) Uso de glóbulos rojos para hematocrito $\geq 24 \%$

c) Uso de noradrenalina (una vez optimizada la precarga), para presión arterial sistólica (PAS) $\geq 100 \mathrm{mmHg}$ y media $($ PAM) $\geq 70 \mathrm{mmHg}$

d) Uso de vasopresina, terlipresina (bolo i.v. 0,25-1 mg), o desmopresina 1-4 $\mu \mathrm{g}(0,25-1 \mathrm{ml}), 1$ a 2 veces al día 


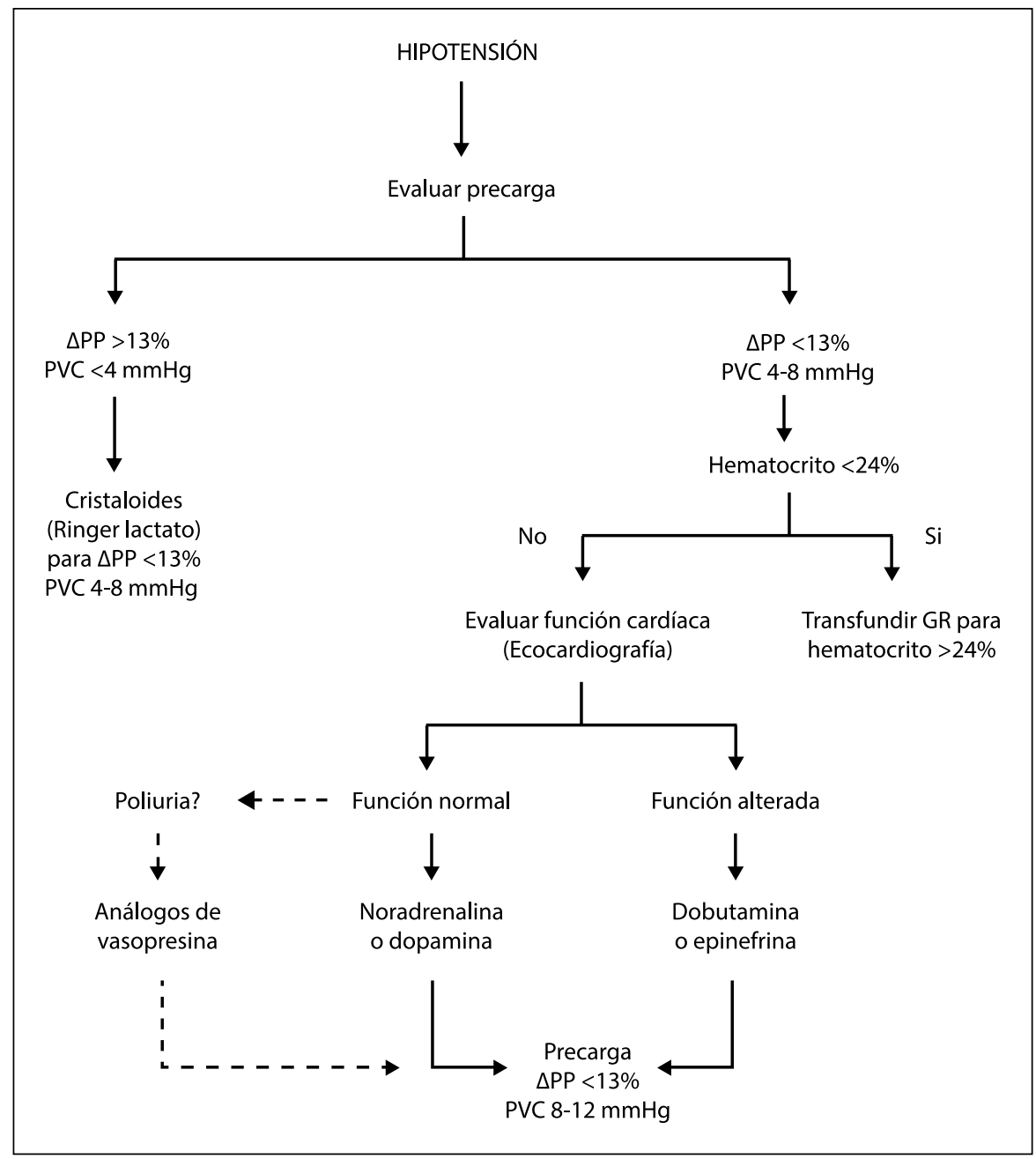

Figura 2. Esquema de manejo de la hipotensión durante la muerte cerebral. Abreviaciones: $G R$, glóbulos rojos; $\triangle P P$, variación en la presión de pulso; PVC, presión venosa central.
Aun así, en un hospital con menos recursos humanos especializados, pensamos que la medición seriada de la PVC puede ser útil ya que un alto porcentaje de los donantes son pacientes sanos o no tienen gran inestabilidad hemodinámica. Este parámetro, incorrectamente utilizado en muchos lugares como indicador de hipovolemia, a la luz de la fisiología resulta ser un buen marcador de disfunción de ventrículo derecho (VD) cuando se eleva. Cada paciente maneja valores de PVC propios a su equilibrio hemodinámico, siendo la mantención de este valor en niveles bajos el mejor indicador de la suficiencia del VD y de todo el sistema circulatorio ${ }^{21}$. Entonces, la gran mayoría de los donantes puede ser manejado sólo con fluidos para optimizar la precarga $(\mathrm{PVC}<10-12 \mathrm{mmHg}) \mathrm{y}$ dosis bajas de vasopresores ${ }^{20,22}$. Esto es importante pues el aporte excesivo de fluidos puede afectar la viabilidad de órganos, particularmente de los pulmones ${ }^{23,24}$.

Con respecto al tipo de fluidos no hay evidencias claras sobre cuál es el más apropiado, pero los cristaloides parecen ser preferibles al uso de coloides en esta condición ${ }^{1}$. La albúmina tiene pocas ventajas en el manejo general del paciente crítico y se ha asociado a mayor mortalidad en la población neurocrítica, por lo que no es recomendable su uso desde antes que el paciente cumpla criterios de muerte encefálica ${ }^{25,26}$. Los almidones tampoco son recomendados pues en diversas situaciones clínicas aumentan la incidencia de disfunción o falla renal ${ }^{5,27,29}$. Incluso las nuevas formulaciones 
de almidones $(130 / 0,4)$ han mostrado mayor tasa de disfunción renal y terapia dialítica en pacientes críticos $^{30,32}$.

La mayoría de las guías actuales sobre manejo del potencial donante recomiendan el uso de glóbulos rojos para obtener un hematocrito sobre $30 \%{ }^{1,6,33}$, pero evidencia específica para este subgrupo no es categórica. Un estudio multicéntrico canadiense en pacientes críticos generales estables no mostró diferencias clínicas al utilizar una estrategia restrictiva de transfusión de glóbulos rojos (hematocrito $21 \%$ ) versus una estrategia liberal (hematocrito 30\%). Incluso hubo menos edema pulmonar y síndrome de distrés respiratorio agudo (SDRA) con la estrategia restrictiva ${ }^{34}$. Más aún, en un reciente estudio en pacientes con trauma cerebral grave, el usar un umbral de transfusión de $21 \%$ vs $30 \%$ se asoció a mejor pronóstico neurológico a 6 meses $^{35}$. En otro aspecto, existe abundante evidencia clínica de que el uso de sangre y hemoderivados puede facilitar el desarrollo de SDRA asociado con la transfusión (TRALI, transfusion-related acute lung injury), teniendo implicancias en la donación de pulmón ${ }^{36,37}$. En ausencia de estudios específicos al respecto, y por ser una población de pacientes jóvenes y con escasa comorbilidad, nos parece razonable fijar un umbral de transfusión bajo $21 \%$, y en caso de inestabilidad hemodinámica intentar mantener un nivel de hematocrito sobre $24 \%{ }^{38}$.

El uso de drogas vasoactivas, dopamina o noradrenalina, es recomendable si no se logra obtener presiones de perfusión sistémicas adecuadas pese a una precarga optimizada ${ }^{39}$. La dopamina no posee un efecto protector sobre la función renal en pacientes críticos ${ }^{40}$, pero sobre 70 a $90 \%$ de los pacientes puede ser manejado exitosamente con una adecuada reanimación con fluidos y dosis bajas de esta droga $(5-10 \mu \mathrm{g} / \mathrm{kg} /$ $\min )^{6,41,42}$. El concepto a destacar es que deben ser dosis bajas. En nuestro grupo, favorecemos el uso de noradrenalina basado en la amplia experiencia con esta droga en pacientes críticos ${ }^{43,44}$. Sin embargo, el uso de dosis moderadas o altas de noradrenalina $(>0,05-0,1 \mu \mathrm{g} / \mathrm{kg} / \mathrm{min})$ se asocia a un aumento en la tasa de disfunción post trasplante cardiaco ${ }^{7,45}$.

En resumen, como en todo paciente crítico, en el potencial donante hay que procurar una presión de perfusión adecuada a todos los órganos, teniendo cuidado con evitar la sobrecarga de fluidos.

\section{Manejo ventilatorio del potencial donante cadáver}

Por definición, los pacientes en muerte encefálica requieren de manipulación de su vía aérea y ventilación mecánica (Tabla 4). Existe abundante evidencia que la ventilación mecánica con altos volúmenes o presiones sobre la vía aérea es capaz de dañar el pulmón, aumentar la respuesta inflamatoria y producir daño a distancia en pacientes con SDRA ${ }^{46,47}$. La ventilación protectora, con el uso de bajo volumen corriente corregido al peso ideal (IBW, ideal body weight), es capaz de atenuar la respuesta inflamatoria y mejorar el pronóstico de estos pacientes ${ }^{47}$.

Diversas guías sugieren el uso de volumen corriente (Vt) hasta 10, ó 10-12 ml/kg para mantener normo a leve hipocapnia y bajos niveles de PEEP para facilitar el drenaje venoso cerebral en el paciente neurocrítico ${ }^{6,33}$. Desgraciadamente, esta ventilación puede aumentar la respuesta inflamatoria y aumentar el daño pulmonar y sistémico ${ }^{2,48}$. Más aún, pocas guías se refieren a la necesidad de corregir este $\mathrm{Vt}$ al peso ideal, lo cual pone en mayor riesgo de daño a mujeres y obesos ${ }^{49}$. Cada vez hay más evidencia que la ventilación con alto volumen corriente también puede producir daño en pacientes con pulmones sanos ${ }^{48,51}$. En un estudio prospectivo observacional en 86 pacientes con trauma cerebral grave, Mascia et al, mostraron que $22 \%$ de ellos desarrolló SDRA durante las primeras $72 \mathrm{~h}$ de ventilación, siendo el uso de un Vt elevado $(>11 \mathrm{ml} / \mathrm{kg}$ IBW) un predictor independiente para el desarrollo de esta complicación ${ }^{50}$. En forma más reciente, Determann et al, demostraron que

\section{Tabla 4. Manejo ventilatorio del donante cadáver}

a) Modalidad: Ventilación controlada por volumen

b) Volumen corriente de 6 a $8 \mathrm{ml} / \mathrm{Kg} \mathrm{IBW}$ *

Titular para presión meseta $<25-30 \mathrm{cmH}_{2} \mathrm{O}$ y presión distensión $<15-20 \mathrm{cmH}_{2} \mathrm{O}$

c) Frecuencia respiratoria para normocapnia $\left(\mathrm{PaCO}_{2} 35\right.$ $50 \mathrm{mmHg}$ )

d) PEEP 5 a $10 \mathrm{cmH}_{2} \mathrm{O}$. Aumentar hasta $15 \mathrm{cmH}_{2} \mathrm{O}$ frente a hipoxemia (trauma torácico, aspiración, etc.) para mantener $\mathrm{FiO}_{2}$ menor a $60 \%$

*IBW, peso ideal ("ideal body weight"). Varones $=50+$ 0,91 (altura $[\mathrm{cm}]-152,4$ ). Mujeres $=45,5+0,91$ (altura $[\mathrm{cm}]-152,4)$ 
un Vt de $10 \mathrm{ml} / \mathrm{kg}$ IBW aumentó el desarrollo de SDRA de $2,6 \%$ a $13,5 \%(\mathrm{p}<0,001)$ en relación al uso de Vt $6 \mathrm{ml} / \mathrm{kg}$ IBW en 150 pacientes sin daño pulmonar $^{51}$. Del mismo modo, en pacientes sometidos a cirugía a cardíaca, Vt superiores a $10 \mathrm{ml} / \mathrm{kg}$ IBW fue un factor de riesgo para el desarrollo de falla de otros órganos ${ }^{49}$. Recientemente, un estudio randomizado multicéntrico que incluyó 400 pacientes sometidos a cirugía abdominal mayor, demostró que una estrategia ventilatoria protectora consistente en limitación del Vt a $8 \mathrm{ml} / \mathrm{kg}$ y uso de PEEP, fue capaz de reducir la incidencia de complicaciones posoperatorias pulmonares y extrapulmonares a la tercera parte ${ }^{52}$.

Finalmente, en un estudio multicéntrico randomizado en 118 pacientes con muerte encefálica, la aplicación de una estrategia ventilatoria protectora (Vt 6-8 ml/kg IBW, PEEP 8-10 $\mathrm{cmH}_{2} \mathrm{O}$ ), vs una convencional (Vt 10-12 ml/kg IBW, PEEP 3-5 cm $\mathrm{H}_{2} \mathrm{O}$ ), aumentó el número de pacientes que cumplió criterios para donación de pulmón de $54 \%$ a $95 \%(p=0,001)^{53}$. Entre los criterios de elegibilidad para donación de pulmón se cuenta una $\mathrm{PaO}_{2} / \mathrm{FiO}_{2}>300$ con $\mathrm{O}_{2} 100 \%$ y $5 \mathrm{cmH}_{2} \mathrm{O}$ PEEP, una radiografía de tórax normal, y una presión máxima de vía aérea menor a $30 \mathrm{cmH}_{2} \mathrm{O}$. La estrategia protectora duplicó el número de pacientes en que se procuró efectivamente los pulmones ( $54 \%$ vs $27 \%, \mathrm{p}=0,004)$. Este estudio recalca la importancia de la ventilación protectora, que debiera ser aplicada en todos los pacientes neurocríticos, incluso aquellos con mal pronóstico vital ${ }^{54}$.

En resumen, la ventilación protectora es mandatoria y fundamental para optimizar los resultados en todos los pacientes críticos, incluido el potencial donante. Frente al escenario del trasplante pulmón la ventilación protectora es particularmente necesaria.

\section{Manejo endocrinológico del potencial donante cadáver}

Múltiples cambios endocrinos han sido descritos en pacientes con muerte encefálica, pero su magnitud es variable y el significado clínico incierto ${ }^{1,13,55,56}$. El uso de hormonas exógenas (fundamentalmente corticoides y hormona tiroidea) no ha demostrado un beneficio claro en la sobrevida del injerto ${ }^{57}$. Más importante parece ser mantener una adecuada perfusión sistémica en el donante cadáver ${ }^{58}$.

La diabetes insípida (DI) ocurre en más de $50 \%$ de los pacientes en muerte encefálica ${ }^{14,16,56,59}$, debido a que la irrigación hipofisiaria posterior depende del flujo de la carótida interna que se compromete como causa o consecuencia de la muerte cerebral, lo que lleva a una depleción precoz de hormona antidiurética $(\mathrm{ADH})^{55}$. La falta de $\mathrm{ADH}$ impide que el túbulo renal reabsorba agua libre, traduciéndose en una poliuria con volúmenes de orina tan altos como $500 \mathrm{ml} /$ hora que, si la reposición no es adecuada, puede llevar a o perpetuar una situación de hipovolemia, hipotensión, hipernatremia y otras alteraciones hidroelectrolíticas ${ }^{1,59}$. La donación de pacientes hipernatrémicos $(\mathrm{Na}>155 \mathrm{mEq} / \mathrm{l})$ se ha asociado a peores resultados en trasplante hepático ${ }^{60}$, si bien estudios más recientes no muestran diferencias en la sobrevida a 1 año en receptores de hígados con marcada hipernatremia ${ }^{61,62}$.

El manejo de la DI es inicialmente sintomático, con soluciones hipotónicas para restaurar la natremia y el volumen intravascular (Tabla 5). El uso de análogos de vasopresina (desmopresina o terlipresina) es fuertemente recomendado en pacientes con hipotensión y poliuria, o en presencia de hipernatremia severa, con el objetivo de lograr una diuresis de entre $100-200 \mathrm{ml} / \mathrm{h}^{38,63,66}$. Si bien ésta es una recomendación ampliamente aceptada,

\section{Tabla 5. Manejo de la diabetes insípida}

Frente a diuresis $>300 \mathrm{ml} / \mathrm{h}$, asociada a hipotensión o hipernatremia severa:

a) Reposición del $50 \%$ de la diuresis obtenidas previamente, con solución Ringer lactato o solución salina isotónica. Es conveniente hacer balances cada 4-6 h.

b) Mantener una volemia o precarga adecuada (PVC 4-8 mmHg o $\triangle \mathrm{PP}<13 \%$ ).

c) Manejo de hipernatremia $(\mathrm{Na}+>150 \mathrm{mEq} / \mathrm{l})$ : - Restricción en aporte de sodio

- Uso de solución salina $0,45 \%$ o solución glucosada $3-5 \%$ en vez de solución salina isotónica, Ringer lactato o para reponer la diuresis

d) Uso de análogos de vasopresina frente a diuresis mayor de $300 \mathrm{ml} / \mathrm{h}$ asociada a hipotensión o hipernatremia:

Desmopresina 1-4 $\mu \mathrm{g}(0,25-1,0 \mathrm{ml}), 1$ a 2 veces al día Terlipresina 0,25-1,0 mg en bolo i.v. 
en un reciente meta-análisis, el uso de desmopresina no mostró ventajas en la función del órgano en receptores de trasplante renal ${ }^{57}$.

También los análogos de vasopresina han sido usado en casos de shock refractario o frente al uso de altas dosis de catecolaminas ${ }^{64}$. En un estudio en 80 donantes de corazón, el uso de vasopresina redujo o eliminó la necesidad de norepinefrina en más de la mitad de los pacientes ${ }^{58}$. Algunas guías clínicas recomiendan la vasopresina como el vasopresor de primera línea en la reanimación del potencial donante, antes incluso que el uso de catecolaminas $^{38}$.

El uso de corticoides en el potencial donante ha sido largamente debatido. Debido al cese del funcionamiento del eje hipotálamo-hipófisis-adrenal, existe una insuficiencia esteroidal absoluta. La literatura destaca los beneficios de su administración en cuanto a mejorar la funcionalidad general de los órganos del donante antes del procuramiento y de la posterior sobrevida del injerto ${ }^{67,68}$. También, se ha reportado que mejora la oxigenación del pulmón procurado, la función cardíaca posttrasplante y atenúa los efectos proinflamatorios de las citoquinas liberadas como consecuencia de la muerte encefálica ${ }^{67,69}$.

La "resucitación hormonal", consistente en la administración de metilprednisolona, vasopresina y hormona tiroidea, resultó en una mejor función del injerto a corto plazo al disminuir la disfunción del órgano post-trasplante ${ }^{68}$. En otro estudio randomizado contra placebo, el uso de 1 g de metilprednisolona disminuyó la inflamación en los donantes, pero no redujo la incidencia de insuficiencia renal post-trasplante ${ }^{70}$.

Pese a no contar con mejor evidencia, la metilprednisolona ( $15 \mathrm{mg} / \mathrm{kg}$, en bolo i.v.) es usada en forma frecuente en el potencial donante con el objeto de modular la respuesta inflamatoria ${ }^{3,13}$. De nuestra experiencia en pacientes críticos, preferimos el uso de esteroides en aquellos pacientes con dosis moderadas o altas de noradrenalina $(>$ $0,1 \mu \mathrm{g} / \mathrm{kg} / \mathrm{min})^{44}$. Otros autores recomiendan el uso de metilprednisolona tan pronto como sea posible debido a que además de sus efectos inmunomodulatorios, presenta pocos efectos adversos y es de bajo costo ${ }^{55}$.

La disfunción hipotalámica también produce pérdida del control de la temperatura e hipotermia $^{1,6}$. El centro de regulación térmica del organismo se encuentra localizado en grupos neuronales del núcleo preóptico del hipotálamo anterior, que recibe información de receptores térmicos cutáneos y en grandes vasos, vísceras y médula espinal, además de la temperatura de la sangre que perfunde el hipotálamo. La hipotermia puede producir arritmias, coagulopatía, disfunción cardíaca, entre otros, y dificulta el diagnóstico de muerte encefálica. La hipotermia es más fácil de prevenir que tratar, por lo que se recomienda el uso de fluidos tibios, humidificación activa de la vía aérea y equipos de calentamiento convectivo.

La hiperglicemia es frecuente y multifactorial: secundaria al estrés, al aumento de los niveles de hormonas contrarreguladoras, al uso concomitante de esteroides, cambios en el metabolismo de carbohidratos, infusión de soluciones que contienen glucosa, y resistencia periférica a la insulina ${ }^{1,6,71,72}$. Algunos autores han mostrado una asociación entre hiperglicemia y disfunción renal post-trasplante ${ }^{73}$. La experiencia de pacientes críticos generales sugiere que un control demasiado estricto de la glicemia (81-108 mg/dL) puede aumentar la mortalidad en relación a un manejo más convencional $(140-180 \mathrm{mg} / \mathrm{dL})^{74,75}$. De este modo, la hiperglicemia $(>180 \mathrm{mg} / \mathrm{dL})$ en el potencial donante cadáver debe ser reconocida y tratada con insulina, preferentemente por vía intravenosa, ya que su absorción por otras vías es variable y de difícil control.

\section{Conclusión}

El manejo médico del potencial donante cadáver es fundamental para aumentar el procuramiento de órganos y optimizar su función posttrasplante. En particular, se debe mantener una adecuada presión de perfusión, teniendo cuidado con evitar la sobrecarga de fluidos, mantener una estrategia protectora de ventilación, y un soporte metabólico y hormonal según la condición clínica de cada paciente. El reconocimiento precoz de los potenciales problemas y complicaciones y su tratamiento oportuno puede mejorar los resultados del trasplante de órganos en los años futuros.

\section{Referencias}

1. McKeown DW, Bonser RS, Kellum JA. Management of the heartbeating brain-dead organ donor. Br J Anaesth 2012; 108 Suppl 1: i96-107. 
2. Mascia L, Mastromauro I, Viberti S, Vincenzi M, Zanello M. Management to optimize organ procurement in brain dead donors. Minerva Anestesiol 2009; 75: 125-33.

3. Venkateswaran RV, Patchell VB, Wilson IC, Mascaro JG, Thompson RD, Quinn DW, et al. Early donor management increases the retrieval rate of lungs for transplantation. Ann Thorac Surg 2008; 85: 278-86; discussion 286.

4. Salim A, Velmahos GC, Brown C, Belzberg H, Demetriades D. Aggressive organ donor management significantly increases the number of organs available for transplantation. J Trauma 2005; 58: 991-4.

5. Robert R, Guilhot J, Pinsard M, Longeard PL, Jacob JP, Gissot V, et al. A pair analysis of the delayed graft function in kidney recipient: the critical role of the donor. J Crit Care 2010; 25: 582-90.

6. Wood KE, Becker BN, McCartney JG, D'Alessandro AM, Coursin DB. Care of the potential organ donor. N Engl J Med 2004; 351: 2730-9.

7. Stehlik J, Feldman DS, Brown RN, VanBakel AB, Russel $\mathrm{SD}$, Ewald GA, et al. Interactions among donor characteristics influence post-transplant survival: a multiinstitutional analysis. J Heart Lung Transplant 2010; 29: 291-8.

8. Feng S, Goodrich NP, Bragg-Gresham JL, Dykstra DM, Punch JD, DebRoy MA, et al. Characteristics associated with liver graft failure: the concept of a donor risk index. Am J Transplant 2006; 6: 783-90.

9. Bellingham JM, Santhanakrishnan C, Neidlinger N, Wai P, Kim J, Niederhaus S, et al. Donation after cardiac death: a 29-year experience. Surgery 2011; 150: 692-702.

10. Detry O, Le Dinh H, Noterdaeme T, De Roover A, Honore P, Squifflet JP, et al. Categories of donation after cardiocirculatory death. Transplant Proc 2012; 44: 118995.

11. Trasplante SCd. [cited Available from: http://www. sociedaddetrasplante.cl/utilidades/biblioteca/section/9guias-clinicas-sociedad-chilena-de-trasplante.html

12. Chile TCd. [cited Available from: http://www.tribunalconstitucional.cl/wp/ver.php?id=397

13. Ranasinghe AM, Bonser RS. Endocrine changes in brain death and transplantation. Best Pract Res Clin Endocrinol Metab 2011; 25: 799-812.

14. Nygaard CE, Townsend RN, Diamond DL. Organ donor management and organ outcome: a 6-year review from a Level I trauma center. J Trauma 1990; 30: 728-32.

15. Oto T, Excell L, Griffiths AP, Levvey BJ, Bailey M, Marasco $\mathrm{S}$, et al. Association between primary graft dysfunction among lung, kidney and heart recipients from the same multiorgan donor. Am J Transplant 2008; 8: 2132-9.
16. Bodenham A, Berridge JC, Park GR. Brain stem death and organ donation. Bmj 1989; 299: 1009-10.

17. Venkateswaran RV, Townend JN, Wilson IC, Mascaro JG, Bonser RS, Steeds RP. Echocardiography in the potential heart donor. Transplantation 2010; 89: 894-901.

18. Szabo G, Hackert T, Sebening C, Vahl CF, Hagl S. Modulation of coronary perfusion pressure can reverse cardiac dysfunction after brain death. Ann Thorac Surg 1999; 67: 18-25; discussion 25-16.

19. Pinsky MR. Hemodynamic evaluation and monitoring in the ICU. Chest 2007; 132: 2020-9.

20. Murugan R, Venkataraman R, Wahed AS, Elder M, Carter M, Madden NJ, et al. Preload responsiveness is associated with increased interleukin- 6 and lower organ yield from brain-dead donors. Crit Care Med 2009, 37: 2387-93.

21. Pinsky MR, Payen D. Functional hemodynamic monitoring. Crit Care 2005, 9: 566-72.

22. López-Navidad A, Caballero F. For a rational approach to the critical points of the cadaveric donation process. Transplant Proc 2001; 33: 795-805.

23. Pennefather SH, Bullock RE, Dark JH. The effect of fluid therapy on alveolar arterial oxygen gradient in braindead organ donors. Transplantation 1993, 56: 1418-22.

24. Angel LF, Levine DJ, Restrepo MI, Johnson S, Sako E, Carpenter A, et al. Impact of a lung transplantation donor-management protocol on lung donation and recipient outcomes. Am J Respir Crit Care Med 2006; 174: 710-6.

25. Myburgh J, Cooper DJ, Finfer S, Bellomo R, Norton R, Bishop N, et al. Saline or albumin for fluid resuscitation in patients with traumatic brain injury. $\mathrm{N}$ Engl J Med 2007; 357: 874-84.

26. Finfer S, Bellomo R, Boyce N, French J, Myburgh J, Norton R. A comparison of albumin and saline for fluid resuscitation in the intensive care unit. $\mathrm{N}$ Engl J Med 2004; 350: 2247-56.

27. Cittanova ML, Leblanc I, Legendre C, Mouquet C, Riou B, Coriat P. Effect of hydroxyethylstarch in brain-dead kidney donors on renal function in kidney-transplant recipients. Lancet 1996; 348: 1620-2.

28. Legendre C, Thervet E, Page B, Percheron A, Noel LH, Kreis H. Hydroxyethylstarch and osmotic-nephrosis-like lesions in kidney transplantation. Lancet 1993; 342: 248 9.

29. Brunkhorst FM, Engel C, Bloos F, Meier-Hellmann A, Ragaller M, Weiler N, et al. Intensive insulin therapy and pentastarch resuscitation in severe sepsis. N Engl J Med 2008; 358: 125-39.

30. Perner A, Haase N, Guttormsen AB, Tenhunen J, Klemenzson G, Aneman A, et al. Hydroxyethyl starch 
130/0.42 versus Ringer's acetate in severe sepsis. N Engl J Med 2012; 367: 124-34.

31. Myburgh JA, Finfer S, Bellomo R, Billot L, Cass A, Gattas D, et al. Hydroxyethyl Starch or Saline for Fluid Resuscitation in Intensive Care. N Engl J Med 2012; 367: 1901-11.

32. Bugedo G, Romero C. Use of hydroxyethyl starch in critically ill patients. Crit Care 2013; 17: 455.

33. Rosengard BR, Feng S, Alfrey EJ, Zaroff JG, Emond JC, Henry ML, et al. Report of the Crystal City meeting to maximize the use of organs recovered from the cadaver donor. Am J Transplant 2002; 2: 701-11.

34. Hebert PC, Wells G, Blajchman MA, Marshall J, Martin C, Pagliarello G, et al. A multicenter, randomized, controlled clinical trial of transfusion requirements in critical care. Transfusion Requirements in Critical Care Investigators, Canadian Critical Care Trials Group. N Engl J Med 1999; 340: 409-17.

35. Robertson CS, Hannay HJ, Yamal JM, Gopinath S, Goodman JC, Tilley BC, et al. Effect of erythropoietin and transfusion threshold on neurological recovery after traumatic brain injury: a randomized clinical trial. JAMA 2014; 312: 36-47.

36. Rana R, Fernández-Pérez ER, Khan SA, Rana S, Winters JL, Lesnick TG, et al. Transfusion-related acute lung injury and pulmonary edema in critically ill patients: a retrospective study. Transfusion 2006; 46: 1478-83.

37. Toy P, Gajic O, Bacchetti P, Looney MR, Gropper MA, Hubmayr R, et al. Transfusion-related acute lung injury: incidence and risk factors. Blood 2012; 119: 1757-67.

38. Shemie SD, Ross H, Pagliarello J, Baker AJ, Greig PD, Brand T, et al. Organ donor management in Canada: recommendations of the forum on Medical Management to Optimize Donor Organ Potential. CMAJ 2006; 174: S13-32.

39. Schnuelle P, Gottmann U, Hoeger S, Boesebeck D, Lauchart W, Weiss C, et al. Effects of donor pretreatment with dopamine on graft function after kidney transplantation: a randomized controlled trial. JAMA 2009; 302: 1067-75.

40. Bellomo R, Chapman M, Finfer S, Hickling K, Myburgh J. Low-dose dopamine in patients with early renal dysfunction: a placebo-controlled randomised trial. Australian and New Zealand Intensive Care Society (ANZICS) Clinical Trials Group. Lancet 2000; 356: 2139-43.

41. Schneider A, Toledo-Pereyra LH, Zeichner WD, Allaben $\mathrm{R}$, Whitten J. Effect of dopamine and pitressin on kidneys procured and harvested for transplantation. Transplantation 1983; 36: 110-1.

42. Schnuelle P, Lorenz D, Mueller A, Trede M, Van Der Woude FJ. Donor catecholamine use reduces acute allograft rejection and improves graft survival after cadaveric renal transplantation. Kidney Int 1999; 56: 738-46.

43. Hernández G, Bruhn A, Romero C, Javier Larrondo F, De La Fuente R, Castillo L, et al. Management of septic shock with a norepinephrine-based haemodynamic algorithm. Resuscitation 2005; 66: 63-9.

44. Castro R, Regueira T, Aguirre ML, Llanos OP, Bruhn A, Bugedo $G$, et al. An evidence-based resuscitation algorithm applied from the emergency room to the ICU improves survival of severe septic shock. Minerva Anestesiol 2008; 74: 223-31.

45. Stoica SC, Satchithananda DK, White PA, Parameshwar J, Redington AN, Large SR. Noradrenaline use in the human donor and relationship with load-independent right ventricular contractility. Transplantation 2004; 78: 1193-7.

46. Ranieri VM, Suter PM, Tortorella C, De Tullio R, Dayer $\mathrm{JM}$, Brienza A, et al. Effect of mechanical ventilation on inflammatory mediators in patients with acute respiratory distress syndrome: a randomized controlled trial. JAMA 1999; 282: 54-61.

47. Ventilation with lower tidal volumes as compared with traditional tidal volumes for acute lung injury and the acute respiratory distress syndrome. The Acute Respiratory Distress Syndrome Network. N Engl J Med 2000; 342: 1301-8.

48. Serpa Neto A, Cardoso SO, Manetta JA, Pereira VG, Esposito DC, Pasqualucci M de O, et al. Association between use of lung-protective ventilation with lower tidal volumes and clinical outcomes among patients without acute respiratory distress syndrome: a meta-analysis. JAMA 2012; 308: 1651-9.

49. Lellouche F, Dionne S, Simard S, Bussieres J, Dagenais F. High tidal volumes in mechanically ventilated patients increase organ dysfunction after cardiac surgery. Anesthesiology 2012; 116: 1072-82.

50. Mascia L, Zavala E, Bosma K, Pasero D, Decaroli D, Andrews $\mathrm{P}$, et al. High tidal volume is associated with the development of acute lung injury after severe brain injury: an international observational study. Crit Care Med 2007; 35: 1815-20.

51. Determann RM, Royakkers A, Wolthuis EK, Vlaar AP, Choi G, Paulus F, et al. Ventilation with lower tidal volumes as compared with conventional tidal volumes for patients without acute lung injury: a preventive randomized controlled trial. Crit Care 2010; 14: R1.

52. Futier E, Constantin J, Jaber S. Protective lung ventilation in operating room: Systematic Review. Minerva Anestesiol 2013.

53. Mascia L, Pasero D, Slutsky AS, Arguis MJ, Berardino 
M, Grasso S, et al. Effect of a lung protective strategy for organ donors on eligibility and availability of lungs for transplantation: a randomized controlled trial. JAMA 2010; 304: 2620-7.

54. Lellouche F, Lipes J. Prophylactic protective ventilation: lower tidal volumes for all critically ill patients? Intensive Care Med 2013; 39: 6-15.

55. Chamorro C, Falcon JA, Michelena JC. Controversial points in organ donor management. Transplant Proc 2009; 41:3473-5.

56. Gramm HJ, Meinhold H, Bickel U, Zimmermann J, von Hammerstein B, Keller F, et al. Acute endocrine failure after brain death? Transplantation 1992; 54: 851-7.

57. Rech TH, Moraes RB, Crispim D, Czepielewski MA, Leitao CB. Management of the brain-dead organ donor: a systematic review and meta-analysis. Transplantation 2013; 95: 966-74.

58. Venkateswaran RV, Steeds RP, Quinn DW, Nightingale P, Wilson IC, Mascaro JG, et al. The haemodynamic effects of adjunctive hormone therapy in potential heart donors: a prospective randomized double-blind factorially designed controlled trial. Eur Heart J 2009; 30: 1771-80.

59. Salim A, Martin M, Brown C, Belzberg H, Rhee P, Demetriades D. Complications of brain death: frequency and impact on organ retrieval. Am Surg 2006; 72: 377-81.

60. Totsuka E, Dodson F, Urakami A, Moras N, Ishii T, Lee $\mathrm{MC}$, et al. Influence of high donor serum sodium levels on early postoperative graft function in human liver transplantation: effect of correction of donor hypernatremia. Liver Transpl Surg 1999; 5: 421-8.

61. Mangus RS, Fridell JA, Vianna RM, Milgrom ML, Chestovich P, Vandenboom C, et al. Severe hypernatremia in deceased liver donors does not impact early transplant outcome. Transplantation 2010; 90: 438-43.

62. Khosravi MB, Firoozifar M, Ghaffaripour S, Sahmeddini MA, Eghbal MH. Early Outcomes of Liver Transplants in Patients Receiving Organs From Hypernatremic Donors. Exp Clin Transplant 2013.

63. Yoshioka T, Sugimoto H, Uenishi M, Sakamoto T, Sadamitsu D, Sakano T, et al. Prolonged hemodynamic maintenance by the combined administration of vasopressin and epinephrine in brain death: a clinical study. Neurosurgery 1986; 18: 565-7.
64. Blasco V, Leone M, Geissler A, Antonini F, Albanese J, Martin C. Impact of terlipressin on renal graft function. Shock 2008; 29: 667-9.

65. Piazza O, Scarpati G, Rispoli F, Iannuzzi M, Tufano R, De Robertis E. Terlipressin in brain-death donors. Clin Transplant 2012; 26: E571-575.

66. Plurad DS, Bricker S, Falor A, Neville A, Bongard F, Putnam B. Donor hormone and vasopressor therapy: closing the gap in a transplant organ shortage. J Trauma Acute Care Surg 2012; 73: 689-94.

67. Follette DM, Rudich SM, Babcock WD. Improved oxygenation and increased lung donor recovery with high-dose steroid administration after brain death. J Heart Lung Transplant 1998; 17: 423-9.

68. Rosendale JD, Kauffman HM, McBride MA, Chabalewski FL, Zaroff JG, Garrity ER, et al. Hormonal resuscitation yields more transplanted hearts, with improved early function. Transplantation 2003; 75: 1336-41.

69. Pratschke J, Kofla G, Wilhelm MJ, Vergopoulos A, Laskowski I, Shaw GD, et al. Improvements in early behavior of rat kidney allografts after treatment of the brain-dead donor. Ann Surg 2001; 234: 732-40.

70. Kainz A, Wilflingseder J, Mitterbauer C, Haller M, Burghuber C, Perco P, et al. Steroid pretreatment of organ donors to prevent postischemic renal allograft failure: a randomized, controlled trial. Ann Intern Med 2010; 153: 222-30.

71. Bugge JF. Brain death and its implications for management of the potential organ donor. Acta Anaesthesiol Scand 2009; 53: 1239-50.

72. Masson F, Thicoipe M, Gin H, de Mascarel A, Angibeau RM, Favarel-Garrigues JF, et al. The endocrine pancreas in brain-dead donors. A prospective study in 25 patients. Transplantation 1993; 56: 363-7.

73. Blasi-Ibáñez A, Hirose R, Feiner J, Freise C, Stock PG, Roberts JP, et al. Predictors associated with terminal renal function in deceased organ donors in the intensive care unit. Anesthesiology 2009; 110: 333-41.

74. Finfer S, Chittock DR, Su SY, Blair D, Foster D, Dhingra $\mathrm{V}$, et al. Intensive versus conventional glucose control in critically ill patients. N Engl J Med 2009; 360: 1283-97.

75. Egi M, Finfer S, Bellomo R. Glycemic control in the ICU. Chest 2011; 140: 212-20. 